\title{
Inferior Turbinate Surgery in Sleep-Disordered Breathing Patients with Nasal Obstruction: Principles and Various Techniques
}

\author{
Ji Ho Choi, MD, PhD ${ }^{1 *}$, Jong Kyu Lee, $\mathrm{MD}^{2 *}$, Seok Hyun Cho, MD, PhD \\ 'Department of Otorhinolaryngology-Head and Neck Surgery, Soonchunhyang University College of Medicine, Bucheon Hospital, Bucheon, Korea \\ 2Jamsil Hana ENT Clinic, Seoul, Korea \\ ${ }^{3}$ Department of Otorhinolaryngology-Head and Neck Surgery, Hanyang University Hospital, Seoul, Korea
}

Received: March 31, 2018

Revised: May 7, 2018

Accepted: May 28, 2018

Correspondence

Seok Hyun Cho, MD, PhD

Department of Otorhinolaryngology-

Head and Neck Surgery,

Hanyang University Hospital,

222-Wangshimni-ro, Seongdong-gu,

Seoul 04763, Korea

Tel +82-2-2290-8583

Fax +82-2-2293-3335

E-mail shcho@hanyang.ac.kr

*These authors contributed equally to this work.

ORCID

Ji Ho Choi

https://orcid.org/0000-0002-5194-930X

Jong Kyu Lee

https://orcid.org/0000-0002-7774-6455

Seok Hyun Cho

https://orcid.org/0000-0001-8218-5894
Sleep-disordered breathing (SDB) is characterized by the intermittent narrowing or collapse of the upper airway, including the nasal cavity, pharynx, and larynx during sleep. Nasal obstruction is one of the most frequent presenting symptoms in SDB patients, and therefore, medical treatments such as saline nasal irrigation, antihistamine, and topical nasal spray are the first recommendation. If the issue is not resolved, surgical treatments for nasal congestion are helpful in order to alleviate nasal obstruction, reduce snoring, and improve positive airway pressure compliance. Inferior turbinate surgery is one of the most commonly performed nasal surgeries (e.g., endoscopic sinus surgery, septoplasty, etc) used to improve nasal obstruction. There are various inferior turbinate surgical methods including electrocautery, laser-assisted turbinoplasty, radiofrequency-assisted turbinoplasty, outfracture, submucous turbinoplasty, partial turbinectomy, and microdebrider-assisted turbinoplasty. Despite the development of these numerous approaches, no clear guidelines exist as yet to help determine the most appropriate modality for any individual patient. This is due to variation in pathophysiology and the degree and extent of the turbinate hypertrophy between patients. Consequently, a comprehensive understanding of these techniques, as well as the preservative concept of functional nasal physiology, is critically important for all surgeons. We propose that the ideal inferior turbinate surgery would meet the following criteria: 1) be less invasive, 2) incorporate remodeling rather than excessive resection, 3) entail a submucosal versus superficial mucosal technique, 4) represent an individually selected technique that is best suited to the patient, and 5) address long term considerations rather than acute symptomatic relief.

Sleep Med Res 2018;9(1):20-25

Key Words Turbinate, Hypertrophy, Surgery, Sleep-disordered breathing, Review.

\section{INTRODUCTION}

Sleep-disordered breathing (SDB) is a broad term for respiratory abnormalities occurring during sleep and a spectrum of partial or complete upper airway obstruction, ranging from simple snoring to severe obstructive sleep apnea syndrome (OSAS). Nasal obstruction is one of the most common complaints among SDB patients. There are various therapeutic options for the improvement of nasal obstruction including saline nasal irrigation, topical nasal spray, medication, and nasal surgery. Of these, conservative or non-surgical therapy (e.g., irrigation, spray, medication, etc.) is primarily recommended. However, if there is no response to conservative treatment, nasal surgery can be scheduled for alleviation of nasal congestion.

Nasal surgery in patients with SDB can be useful not only in improving nasal obstruction but also in order to decrease snoring [1]. In addition to nasal surgery, it can be helpful to use positive airway pressure (PAP) [2,3]. Nasal congestion in SDB patients with PAP therapy is known to be associated with mouth leak, unintentional mask removal, and the increase of optimal PAP level. Previously, we reported a case of increased PAP compliance after nasal surgery [3]. As a result, nasal surgery can improve poor PAP compliance and reduce the optimal level 
of PAP [2,3].

Inferior turbinate surgery is one of the most frequently used nasal surgeries in patients with nasal obstruction. Inferior turbinate surgery should only be performed by surgeons with a comprehensive understanding of the physiology and anatomy of the nose and turbinates. Olfaction, respiration and protection are the three main functions of nose, with the lateral nasal turbinates (superior, middle, and inferior) serving important roles [4]. Of these, the inferior turbinate is the most important, as it is the largest and contains a highly developed venous plexus that often engorges and becomes hyper-activated. The specific role of the inferior turbinate is threefold: 1) it determines the direction of the inspired nasal airflow, 2) it warms and humidifies the inspired nasal airflow to the optimal conditions for the lower respiratory tract, and 3) it filters the inspired air of potentially hazardous material, such as bacteria, viruses, and dust particles $[4,5]$.

Structurally, inferior turbinate hypertrophy can be subdivided into 3 types: 1) mucosal hypertrophy, 2) osseous hypertrophy, and 3) mixed hypertrophy. Of these, mucosal hypertrophy presents in patients with chronic inflammatory diseases of the nasal cavity, such as allergic rhinitis. Conversely, osseous hypertrophy is often seen in patients with unilateral inferior turbinate hypertrophy secondary to septal deviation [6-8]. Lastly, mixed type hypertrophy is believed to result from both factors described above. A perception of the components of turbinate enlargement provides the guidance needed for rhinologists when deciding on the best surgical method.

Over the years, evolution and development of new surgical devices has led to a number of different surgical techniques used to address inferior turbinate hypertrophy, with many reports extoling the efficacy of each newly developed approach. However, there still remains no clear consensus in the literature regarding the optimal operative technique for inferior turbinate hypertrophy [9-12].

Despite this controversy regarding gold standard therapy, the clear goals of inferior turbinate surgery are to improve nasal obstruction, maintain nasal function, and minimize complications. As such, a comprehensive understanding of pros and cons in each surgical technique is necessary in order to perform optimal inferior turbinate surgery.

The current review will examine a case of nasal surgery including inferior turbinate surgery performed on a SDB patient with low PAP compliance [3]. Subsequently, there will be a summary of key concepts, indications, guidelines, and the most widely used operative techniques for inferior turbinate surgery: electrocautery, laser-assisted turbinoplasty (LAT), radiofrequency-assisted turbinoplasty (RFAT), outfracture, submucous turbinoplasty, partial turbinectomy, and microdebrider-assisted turbinoplasty (MAT).

\section{CASE}

A 47-year-old male adult visited a tertiary referral hospital with SDB symptoms such as snoring, sleep apnea, nasal obstruction, and excessive daytime sleepiness (Epworth Sleepiness Scale = 11). His Body Mass Index was $25.7 \mathrm{~kg} / \mathrm{m}^{2}$ (height was $165 \mathrm{~cm}$ and weight was $70 \mathrm{~kg}$ ). The palatine tonsils size was grade 1 (hidden behind tonsillar pillars) and the palate-tongue positions level was grade 3 (visualization of the soft palate but not the uvula). Upon endoscopic examination of nasal cavity, the nasal septum was deviated to right side and the bilateral inferior turbinate was enlarged. He was diagnosed with severe OSAS according to polysomographic examinations (ApneaHypopnea Index $=43.0$ events/hour, lowest arterial oxygen saturation $=71 \%$, snoring $=37.9 \%$ ). PAP therapy was recommended for his OSAS. Conservative treatment including nasal irrigation, topical steroid, and oral medications was attempted for his nasal obstruction. PAP compliance for the first six months was relatively low (5.4\% for the 1 st three months, $28 \%$ for 2 nd month, and 30.4\% for the 3rd two months). He repeatedly complained of mouth leak and/or unintentional mask removal due to nasal obstruction. The reason for low compliance was thought to be his nasal obstruction. Therefore, inferior turbinate surgery and septoplasty was scheduled and performed. After the surgery, nasal obstruction was alleviated, and PAP compliance for six months was significantly improved $(72.0 \%$ for the 1 st three months and $86.7 \%$ for the 2 nd three months). In addition, the optimal level of PAP was significantly decreased by approximately $1.5 \mathrm{cmH}_{2} \mathrm{O}$ after the nasal surgery.

\section{KEY CONCEPTS}

Patients must be informed that turbinate surgery may not always control the symptoms completely and permanently, and that relapse may be possible at a later date due to its pathophysiological nature. Preoperatively, a bilateral inspection of the nares and a characterization of the major obstruction site in the nasal cavity should be confirmed, while taking into account that the nasal cycle may act as a confounding factor [13]. Age factor is also important and must be accounted for before scheduling the surgery since pediatrics is a growth period and geriatrics are apt to have low nasal functions $[14,15]$.

In most cases, a more conservative approach (rather than an excessive inferior turbinate resection) is optimal because as excessive nasal cavity space after surgery does not necessarily produce better results given the negative effect on nasal physiology $[16,17]$. Additionally, with more conservative approaches, mucociliary function is maintained resulting in better physiologic outcomes $[4,18]$.

Complications of turbinate surgery can include bleeding, crusting, and nasal pain. Most of all, surgeons should be par- 
ticularly attentive of atrophic rhinitis or empty nose syndrome (ENS), a permanent functional impairment that occurs after excessive resection or destruction of the nasal mucosa [16,17]. Thus, to achieve good outcomes after inferior turbinate surgery, it is important for ENT surgeons to have an understanding of nasal function conservation, in which surgery should be conducted regarding surgical modifications of the nasal pathway rather than having an inclination to remove much of the turbinate structure.

\section{INDICATIONS}

The primary indication for inferior turbinate surgery is symptomatic nasal obstruction that occurs with inferior turbinate hypertrophy (secondary to various causes), which restricts airflow via a narrowing of the nasal airway. In such cases, conservative medical therapy, including nasal irrigation, antihistamines, decongestants and intranasal topical steroids, is considered as the initial treatment. However, inferior turbinate surgery should be considered in patients who do not initially respond to these agents. In patients with allergic rhinitis, surgery may also be indicated if symptoms are refractory to conservative management with medications and immunotherapy or if the hypertrophic change of the inferior turbinate is perceived as irreversible.

Preoperatively, the upper airway should always be reviewed, as many other etiologies for nasal obstruction exist, including structural deformities of the nasal septum, sinusitis, nasal polyps, and tumor and adenoids in pediatric patients.

It is also important to differentiate the cause of the turbinate enlargement when choosing a good surgical technique. The topical decongestant test can help to differentiate the components of the obstruction by comparing the change in symptoms, endoscopic findings, and acoustic rhinometry before and after the decongestant is administered. In this way, the inferior turbinate hypertrophy can be classified into 3 types: 1) mucosal hypertrophy, 2) osseous hypertrophy, and 3) mixed hypertrophy [6-8].

\section{GUIDELINES: MULTIMODALITY AND INDIVIDUALIZATION}

The underlying concept of turbinate surgery is increased nasal airway volume better facilitate functional nasal airflow. In deciding the most appropriate operative technique, three factors should be considered: 1 ) the septal condition related to the turbinate, 2) the pathophysiologic state of the inferior turbinate, and 3) surgeon's preference. In cases of clear septal deviation resulting in nasal obstruction, septoplasty is commonly performed prior to the turbinate surgery. But, since the best time for turbinate mucosa manipulation is just after the local infiltrative anesthesia, and especially in RFAT, the inferior tur- binate surgery may be performed prior to septoplasty.

Because each surgical approach requires different tissue manipulation, it is critical for surgeons to understand the underlying pathology of hypertrophic inferior turbinates when deciding the surgical technique. Generally, it is more appropriate to address any mucosal hypertrophy before touching osseous hypertrophy.

Depending on the location and degree of mucosal lesions, multiple modalities [laser, radiofrequency (RF) ablation, and microdebrider] having individual strength and weaknesses could be used solitarily or combined, aiming for the best results.

If these are not sufficient, we can consider the next step to deal with the osseous component of inferior turbinate. At this time, the density and location of the osseous hypertrophy should be taken into consideration before doing bone work. In cases of low bone density, outfracture alone often yields good results, however in cases with severe osseous hypertrophy, submucous turbinoplasty is often more effective and thus is the preferred technique. Additionally, we can use the combination technique by lateral fracturing of remaining low density bony portion after removal of localized high-density lesion via submucosal resection. In cases of allergic rhinitis, the inferior turbinate is considered to be the primary cause for most associated symptoms, including rhinorrhea, sneezing, itching and especially nasal obstruction [18]. As such, inferior turbinate surgery preserving the innate physiologic function is now widely used in order to alleviate symptoms especially for nasal obstruction.

\section{SURGICAL TECHNIQUES}

\section{Electrocautery}

Electrocautery may be applied either to the mucosa or submucosa, with bipolar cautery being preferred over monopolar devices. Surface cautery-performed via either a wire or needle electrode-is used to streak the turbinate mucosa and induce coagulation necrosis, ultimately resulting in sclerotic fibrous connective tissue and a reduction in turbinate size [19]. Conversely, submucosal cautery can also be performed by placing the electrode in the submucosa in order to generate heat and induce fibrosis and wound contracture, which later will also result in volume reduction. Drawbacks associated with this modality include significant injury to the surrounding tissue and damage to the overlying mucosa secondary to excessive heat (up to $800^{\circ} \mathrm{C}$ ) [19]. Other disadvantages of electrocautery include the lack of nasal function preservation and poor longterm outcomes when compared with other surgical techniques $[19,20]$.

\section{Laser-assisted turbinoplasty}

Currently, six different laser systems are being used for inferior turbinate surgery: 1) carbon dioxide (CO2), 2) diode, 3) neo- 
dymium-yttrium aluminum garnet (Nd:YAG), 4) potassiumtitanyl phosphate (KTP), 5) argonion (Ar), and 6) holmiumyttrium aluminum garnet (Ho:YAG). As argon and KTP lasers produce light that is absorbed by endogenous chromophores such as hemoglobin and melanin, the associated coagulation depth is maximized at around $2 \mathrm{~mm}$. Conversely, diode and Nd:YAG lasers are poorly absorbed by water, tissue proteins, and blood, enabling energy transmission to deeper tissue layers. Meanwhile, $\mathrm{CO} 2$ land Ho:YAG lasers show a tendency to be absorbed by water. The $\mathrm{CO} 2$ laser is now the most widely used in inferior turbinate surgery and, the Ho:YAG laser provides a better coagulation capability for improved hemostasis [21].

The underlying concept of LAT is to achieve turbinate volume and surface reduction without excessive damage to surrounding tissue that could result in excessive mucosal loss and/ or bone exposure. Not only can laser surgery be performed in a linear, anterior-to-posterior stripe, or cross-hatching method, treatment can also be applied to either the entire turbinate or a particular focus, such as the anterior turbinate head or inferior edge [21-23].

Certain safety measures are necessary during all laser surgeries: providers must wear protective glasses; the patient's exposed face must be covered with wet cloth; and any gas formed during laser surgery must be removed.

LAT has been reported to have success rates greater than 50\% in relieving nasal symptoms (i.e. nasal obstruction), though decreased mucosal function and mucociliary clearance secondary to heat destruction remains a noteworthy problem [22-24]. To circumvent such side effects, more conservative approaches such as focal treatment areas, lower energies and submucosal laser channeling via an 18 gauge needle (KTP and diode) are recommended.

\section{Radiofrequency-Assisted Turbinoplasty}

The RF generators produce output frequencies in the electromagnetic spectrum that vary from $100 \mathrm{kHz}$ to $4000 \mathrm{kHz}$. These devices allow the temperature of target tissues to be maintained between $60-90^{\circ} \mathrm{C}$ using relatively low energies, thus avoiding damage to the surrounding tissue [25]. While there are monopolar and bipolar methods in RFAT, a bipolar approach is now preferred, as the shorter treatment times allows the procedure to be easier and more controllable. Furthermore, some devices do not require saline use, and some are secured with a feedback function that limits the energy delivery to a certain level $[26,27]$.

The energy produced by RF devices agitates tissue ions and induces coagulative necrosis of the submucosa, so that the subsequent wound healing process results in scar formation and tissue retraction, leading to an overall reduction in size and stiffening of the treated area [22,25,27].

After local anesthesia is administered, a probe is inserted into the anterior portion of the inferior turbinate (parallel to the turbinate structure) as deeply as possible. For additional precision in localization, this may also be performed with endoscopic guidance. Energy delivery is usually conducted at about 3 points at the turbinate during expulsion of the inserted probe. Moreover, three key points need to be considered when delivering energy through the inserted probe: 1) penetration of the mucosa with probe should be avoided; 2) excessive energy delivery should be avoided to prevent unnecessary tissue loss; and 3) bony exposure may occur due to mucosal necrosis secondary to thermal injury of the mucosa.

RFAT has been reported to significantly alleviate all symptoms (e.g., nasal obstruction, rhinorrhea, sneezing, and nasal itching) in patients with allergic rhinitis. Moreover, these effects were reported to persist up to five years after treatment, indicating a good long-term efficacy [28]. Advantages of RFAT include: 1) it can be performed in-office under local anesthesia; 2) its demonstrated safety with low morbidity; 3 ) it requires no nasal packing; 4) it has little risk of mucosa destruction, thus preserving the mucociliary function; and 5) it may be performed selectively in a pediatric population.

Complications include mild postoperative pain that typically resolves spontaneously and nasal obstruction secondary to crusting and mucosal swelling that often resolves after 2 to 3 weeks [29].

\section{Outfracture}

Of all the turbinate surgical techniques that address the osseous component, outfracture (lateralization) of the turbinate is regarded as the most conservative and simplest method, having a significant and proven efficacy. Outfracture is primarily indicated in cases of osseous or mixed hypertrophy [29].

After local anesthesia is administered, a boies elevator is used to fracture and displace the turbinate in a lateral fashion. However, this procedure can be performed in several different ways, depending on the degree of turbinate bone density. In cases with lower density, the inferior turbinate is simply fractured and pushed in a medial to lateral direction. However, in high density cases, the inferior turbinate is first fractured in a lateral to medial direction, after which the turbinate is displaced to a lateral position.

When performed as a monotherapy, the outfracture technique may result in insufficient nasal patency, especially in cases of mixed hypertrophy. Additionally, the turbinate may also return to its initial position over time [5,30,31].

\section{Submucous Turbinoplasty}

For submucous turbinoplasty, an incision is first made at the anterior margin of the inferior turbinate with a No. 15 blade. The resulting mucosal flap surrounding the turbinate bone is then raised, and the hypertrophic bone is resected with forceps in an anterior to posterior direction. As the overlying mucosa is preserved, good mucociliary function is maintained, postoperative complications are rare, and effective symptom relief is 
achieved [20,24,32].

Since most cases of inferior turbinate hypertrophy are mixed type, submucous turbinoplasty is usually performed in tandem with other procedures in order to address the mucosal component.

\section{Partial Turbinectomy}

Partial turbinectomy represents the most classic and primitive methods for inferior turbinate surgery. Although the use of partial turbinectomy is shrinking in clinical practice due to the better efficacy of newer techniques and devices, it remains effective in certain cases [23,33]. Turbinectomy is performed using angled (turbinate) scissors to partially resect the most hypertrophied anterior portion of the turbinate, allowing selective removal of the mucosa and/or the underlying bone.

Maintaining the surface mucosa via approximation around the exposed bone is critically important to reduce complications related to bony exposure, including bone bleeding and infection [21]. Additional care should be taken when performing partial turbinectomy as overzealous resection may lead to excessive bleeding and/or atrophic rhinitis or ENS $[33,34]$.

\section{Microdebrider-Assisted Turbinoplasty}

MAT is one of the most recently developed techniques reported in the literature. Initially, an approximately $0.5 \mathrm{~cm}$ incision is made with a No. 15 blade at the anterior most end of the inferior turbinate, after which a pocket is created by elevating the mucosal flap onto the submucosa. The microdebrider tip is then inserted into the pocket and the hypertrophic submucosal tissue and/or bone is carefully debulked with additional care taken to avoid perforating the mucosal flap [35]. In cases which a polypoid lesion is discovered on the mucosal surface, microdebrider debulking could be used with safety. At this time, special care must be taken in order to prevent excessive tissue removal.

Since the superficial mucosa is preserved, mucociliary function remains in good condition and postoperative complications, including bleeding, crusting and synechiae, are rare. Furthermore, this technique also has been shown to produce prompt and durable symptom relief [36].

\section{CONCLUSION}

Nasal congestion is one of the most frequent complaints among patients with SDB. Nasal surgery can be helpful not only to decrease snoring in SDB patients but also as a way to improve poor PAP compliance in SDB patients with PAP treatment.

Given that inferior turbinates play a crucial role in nasal function, turbinate surgery should be handled carefully and meticulously. The ideal inferior turbinate surgical technique should effectively reduce tissue volume, preserve nasal function, and avoid complications. However, no clear consensus currently ex- ists in the literature regarding the most optimal method for surgical treatment of inferior turbinate hypertrophy.

The authors contend that the ideal surgery would meet the following criteria: 1) be the least invasive, 2) incorporate remodeling rather than excessive resection, 3) entail a submucosal versus superficial mucosal technique, 4) represent an individually selected method that is best suited for the patient, and 5) address long term considerations rather than acute symptomatic relief.

\section{Acknowledgments}

This work was supported by the Bio \& Medical Technology Development Program of the National Research Foundation (NRF) \& funded by the Korean government (MIST) (No. 2017M3A9E2064631)

\section{Conflicts of Interest}

The authors have no financial conflicts of interest.

\section{Authors' Contribution}

Conceptualization: Cho SH, Choi JH. Data curation: Choi JH, Lee JK. Formal analysis: Choi JH, Lee JK, Cho SH. Funding acquisition: Cho SH. Methodology: Choi JH, Lee JK, Cho SH. Project administration: Cho SH. Writing-original draft: Choi JH, Lee JK. Writing_review \& editing: Choi JH, Cho SH.

\section{REFERENCES}

1. Choi JH, Kim EJ, Kim YS, Kim TH, Choi J, Kwon SY, et al. Effectiveness of nasal surgery alone on sleep quality, architecture, position, and sleepdisordered breathing in obstructive sleep apnea syndrome with nasal obstruction. Am J Rhinol Allergy 2011;25:338-41.

2. Poirier J, George C, Rotenberg B. The effect of nasal surgery on nasal continuous positive airway pressure compliance. Laryngoscope 2014; 124:317-9.

3. Jung JH, Seon SW, Hong SN, Choi JH. A case of nasal surgery for a positive airway pressure-intolerant OSAS patient due to nasal obstruction. Sleep Medicine and Psychophysiology 2016;23:97-9.

4. Pohunek P. Development, structure and function of the upper airways. Paediatr Respir Rev 2004;5:2-8.

5. Hol MK, Huizing EH. Treatment of inferior turbinate pathology: a review and critical evaluation of the different techniques. Rhinology 2000; 38:157-66.

6. Farmer SE, Eccles R. Chronic inferior turbinate enlargement and the implications for surgical intervention. Rhinology 2006;44:234-8.

7. Berger G, Gass S, Ophir D. The histopathology of the hypertrophic inferior turbinate. Arch Otolaryngol Head Neck Surg 2006;132:588-94.

8. Akoğlu E, Karazincir S, Balci A, Okuyucu S, Sumbas H, Dağli AS. Evaluation of the turbinate hypertrophy by computed tomography in patients with deviated nasal septum. Otolaryngol Head Neck Surg 2007; 136:380-4.

9. Batra PS, Seiden AM, Smith TL. Surgical management of adult inferior turbinate hypertrophy: a systematic review of the evidence. Laryngoscope 2009;119:1819-27.

10. Clement WA, White PS. Trends in turbinate surgery literature: a 35year review. Clin Otolaryngol Allied Sci 2001;26:124-8.

11. Bhandarkar ND, Smith TL. Outcomes of surgery for inferior turbinate hypertrophy. Curr Opin Otolaryngol Head Neck Surg 2010;18:49-53.

12. Leong SC, Eccles R. Inferior turbinate surgery and nasal airflow: evidence-based management. Curr Opin Otolaryngol Head Neck Surg 2010;18:54-9.

13. Lang C, Grützenmacher S, Mlynski B, Plontke S, Mlynski G. Investigating the nasal cycle using endoscopy, rhinoresistometry, and acous- 
tic rhinometry. Laryngoscope 2003;113:284-9.

14. Kim SW, Mo JH, Kim JW, Kim DY, Rhee CS, Lee CH, et al. Change of nasal function with aging in Korean. Acta Otolaryngol Suppl 2007;127 (sup558):90-4.

15. Jiang ZY, Pereira KD, Friedman NR, Mitchell RB. Inferior turbinate surgery in children: a survey of practice patterns. Laryngoscope 2012; 122:1620-3.

16. Chhabra N, Houser SM. The diagnosis and management of empty nose syndrome. Otolaryngol Clin North Am 2009;42:311-30.

17. Houser SM. Surgical treatment for empty nose syndrome. Arch Otolaryngol Head Neck Surg 2007;133:858-63.

18. Chhabra N, Houser SM. The surgical management of allergic rhinitis. Otolaryngol Clin North Am 2011;44:779-95.

19. Salzano FA, Mora R, Dellepiane M, Zannis I, Salzano G, Moran E, et al. Radiofrequency, high-frequency, and electrocautery treatments vs partial inferior turbinotomy: microscopic and macroscopic effects on nasal mucosa. Arch Otolaryngol Head Neck Surg 2009;135:752-8.

20. Passàli D, Passàli FM, Damiani V, Passàli GC, Bellussi L. Treatment of inferior turbinate hypertrophy: a randomized clinical trial. Ann Otol Rhinol Laryngol 2003;112:683-8.

21. Chang CW, Ries WR. Surgical treatment of the inferior turbinate: new techniques. Curr Opin Otolaryngol Head Neck Surg 2004;12:53-7.

22. Rhee CS, Kim DY, Won TB, Lee HJ, Park SW, Kwon TY, et al. Changes of nasal function after temperature-controlled radiofrequency tissue volume reduction for the turbinate. Laryngoscope 2001;111:153-8.

23. Sapçi T, Sahin B, Karavus A, Akbulut UG. Comparison of the effects of radiofrequency tissue ablation, $\mathrm{CO} 2$ laser ablation, and partial turbinectomy applications on nasal mucociliary functions. Laryngoscope 2003;113:514-9.

24. Cassano M, Granieri C, Del Giudice AM, Mora F, Fiocca-Matthews E, Cassano P. Restoration of nasal cytology after endoscopic turbinoplasty versus laser-assisted turbinoplasty. Am J Rhinol Allergy 2010;24:310-4.

25. Li KK, Powell NB, Riley RW, Troell RJ, Guilleminault C. Radiofrequency volumetric tissue reduction for treatment of turbinate hypertrophy: a pilot study. Otolaryngol Head Neck Surg 1998;119:569-73.

26. Hytönen ML, Bäck LJ, Malmivaara AV, Roine RP. Radiofrequency thermal ablation for patients with nasal symptoms: a systematic review of effectiveness and complications. Eur Arch Otorhinolaryngol 2009; 266:1257-66.

27. Cavaliere M, Mottola G, Iemma M. Monopolar and bipolar radiofrequency thermal ablation of inferior turbinates: 20-month follow-up. Otolaryngol Head Neck Surg 2007;137:256-63.

28. Lin HC, Lin PW, Friedman M, Chang HW, Su YY, Chen YJ, et al. Longterm results of radiofrequency turbinoplasty for allergic rhinitis refractory to medical therapy. Arch Otolaryngol Head Neck Surg 2010;136: 892-5.

29. Aksoy F, Ylldırım YS, Veyseller B, Ozturan O, Demirhan H. Midterm outcomes of outfracture of the inferior turbinate. Otolaryngol Head Neck Surg 2010;143:579-84.

30. Goode RL. Surgery of the turbinates. J Otolaryngol 1978;7:262-8.

31. Marquez F, Cenjor C, Pastormerlo G. Multiple submucosal out-fracture of the inferior turbinate: a surgical technique for inferior turbinate hypertrophy. Rhinology: A State of the Art: Proceedings Pf the XVth Congress of the European Rhinologic Society and the XIIIth International Symposium on Infection and Allergy of the Nose, Coppenhagen, Denmark, June 19-23, 1994: Kugler Publications;1995. p.103.

32. Mabry RL. Inferior turbinoplasty: patient selection, technique, and long-term consequences. Otolaryngol Head Neck Surg 1988;98:60-6.

33. Garzaro M, Landolfo V, Pezzoli M, Defilippi S, Campisi P, Giordano C, et al. Radiofrequency volume turbinate reduction versus partial turbinectomy: clinical and histological features. Am J Rhinol Allergy 2012; 26:321-5.

34. Martinez SA, Nissen AJ, Stock CR, Tesmer T. Nasal turbinate resection for relief of nasal obstruction. Laryngoscope 1983;93:871-5.

35. Lee DH, Kim EH. Microdebrider-assisted versus laser-assisted turbinate reduction: comparison of improvement in nasal airway according to type of turbinate hypertrophy. Ear Nose Throat J 2010;89:541-5.

36. Cingi C, Ure B, Cakli H, Ozudogru E. Microdebrider-assisted versus radiofrequency-assisted inferior turbinoplasty: a prospective study with objective and subjective outcome measures. Acta Otorhinolaryngol Ital 2010;30:138-43. 